\title{
TCP Friendly Rate Adaptation for Multimedia Streaming in Mobile ad hoc Networks
}

\author{
Zhenghua Fu, Xiaoqiao Meng, and Songwu Lu \\ Computer Science Department, University of California, Los Angeles \\ $\{$ zfu, xqmeng, slu\}@cs.ucla.edu
}

\begin{abstract}
Transport protocol for supporting multimedia streaming in mobile ad hoc networks has to cope with the rich dynamics, such as mobility-induced disconnection and reconnection, high out-of-order delivery ratios, channel errors, and network congestion. In this work, we design and implementate ADTFRC, a TCPfriendly transport protocol for ad hoc networks. ADTFRC adapts wireline TFRC protocol to ad hoc networks with improved rate adaptation behavior, the capability of application layer framing and selective retransmission. ADTFRC detects different packet loss behaviors based on end-to-end measurements of multiple metrics. This allows ADTFRC to more accurately gauge the network behavior and achieve higher throughput. Simulations show that the performance of ADTFRC is higher than standard TFRC and TCP with explicit-link-failure-notification (ELFN) support in terms of throughput, rate adaptation behavior and application level quality while still maintaining the TCP-Friendliness property.
\end{abstract}

\section{Introduction}

The new generation wireless networks offer much higher transmission rate, e.g., IEEE 802.11b supports up to $11 \mathrm{Mbps}$ transmission rate, IEEE 802.11a can support even up to $54 \mathrm{Mbps}$. It therefore feasible to run multimedia applications over mobile ad hoc networks. Such applications can include multimedia instant messaging, environmental monitoring, distributed gaming and etc. In this paper, we study protocol design to support multimedia streaming over ad hoc networks. Most of the relevant research efforts are focused on the low-layer design such as service differentiation in MAC QoS-aware routing, admission control and adaptive packet scheduling. Different from these work, we address the transport-layer issues. Specifically, we adapt the popular, slowly responsive congestion control protocol - TCP Friendly Rate Control (TFRC), which is originally proposed for wired multimedia transport, to mobile ad hoc networks.

Early proposals use UDP to carry multimedia streams [20]. Since UDP does not provide congestion control, unresponsive multimedia flows will compete unfairly with other responsive TCP flows. Using TCP in multimedia streaming transport can prevent congestion collapse, however, TCP provides $100 \%$ reliability through its retransmission mechanism, which is not necessary for loss-tolerant multimedia streaming. Moreover, TCP halves its transmission rate upon any congestion event; such dramatic rate oscillations are deemed to be detrimental to multimedia applications.

Recent research on multimedia transport design has focused on developing a TCP friendly protocol that does not react to any single congestion event dramatically but 
slowly adapts to network dynamics [16][18]. A noticeable proposal is TCP Friendly Rate Control (TFRC) [17][19], which calculates a TCP friendly throughput and increases/decreases the transmission rate accordingly.

There are several technical challenges for TFRC to function well in mobile ad hoc networks. It is well known that because ad hoc networks exhibit a rich set of packet loss behaviors, directly applying the congestion control mechanisms of TFRC upon every packet loss typically leads to unsatisfactory performance [1][6][7][5]. Moreover, TFRC has to handle several ad hoc networks specific network events, i.e., mobilityinduced disconnection and re-connection, route change induced out-of-order delivery and error/contention-prone wireless transmission 1 . These events require TFRC to respond differently from congestion control. For example, it makes more sense to ignore a random packet loss incurred by channel errors rather than to multiplicatively decrease the sending rate [3]; and it seems to be more appropriate to periodically probe the network during disconnections for a prompt recovery than to slow down and exponentially increase the retransmission timer [1]. Another challenge for TFRC is to accurately detecting and differentiating all these network events. Packet loss as the only detector used by conventional TCP/TFRC flows has been shown to fail to differentiate all these new events [8].

\subsection{Rate Adaptation in ad hoc Networks}

In order to effectively adapt the transmission rate, network congestion should be reliably detected. In particular, among all kinds of packet losses, the congestion loss probability needs to be estimated; treating all losses as congestion loss leads to undesirable rate adaptations. Most of the literature on congestion detection for ad hoc networks endorses a network-oriented approach. In this approach, the routers implement a monitoring module and generate explicit notifications to send back to the TCP sender upon various packet losses. Specifically, if mobility triggers network disconnection, an explicit link failure notification (ELFN) will be sent to the sender [1]; if a congestion loss occurs, an explicit congestion notification (ECN) message is generated [23]; if the router observes a packet loss induced by the wireless channel [3] [4], an explicit loss notification (ELN) will be sent to the TCP sender. Although such a network-oriented approach is can effectively improve TCP performance, it suffers from several drawbacks when it is used for rate adaptation in ad hoc networks.

First, the failure notifications, such as ELFN, generated by intermediate nodes are directly sent to the sender without the awareness of the receiver. Hence, a single packet loss event can trigger two different reports, one is from intermediate nodes and the other comes from the receiver. Because the two reports can arrive at the sender in an arbitrary order and at different time instances, it is difficult to combine the two observed reports and render a consistent image of the overall network condition 2 . Secondly, it is difficult

\footnotetext{
${ }^{1}$ Even with link-layer retransmissions of 802.11 MAC, packet loss still occurs due to bursty channel error or MAC-layer contentions.

${ }^{2}$ Even if the measurements are performed at sender side, feedbacks from both receiver and intermediate nodes are still needed, same complication arises when trying to combine the two overlapping observations.
} 
to globally deply monitoring modules at every node because of the heterogeneity of an ad hoc environment.

Based on the above considerations, an end-to-end approach is more desirable for TCP-Friendly rate adaptation in mobile ad hoc networks since it naturally falls into the existing TFRC protocol [17]. An end-to-end approach means that the TCP receiver differentiates network events, decides the occurrence of congestion and sends feedback to the sender. The sender then adjusts the transmission rate accordingly. Compared with the network-oriented approach, such an end-to-end approach is much easier to be implemented and deployed in practice.

We notice a relevant work [24] which uses packet out-of-order to differentiate packet losses due to route changes or network congestion. However, [24] assumes that packet losses can only be caused by route changes or congestion. Our contribution is that we provide a more general and reliable solution to detect network conditions while still preserving the end-to-end principle.

\subsection{Main Contributions}

The key innovation proposed in this paper is the use of multi-metric joint detection instead of single-metric detection. Because end-to-end measurement data in ad hoc networks are usually highly noisy, frequent false identifications and notifications can happen [8]. How to robustly detect events through noisy measurements imposes a challenge to the design. Based on a multi-metric joint detection, we exploit the degree of independence in the measurement noise of each individual metrics, so that the probability of false identification is significantly reduced by cross verification among the multiple metrics.

In addition, we applied the Application Level Framing (ALF) and Partial Reliability techniques to improve the quality of the multimedia streaming perceived by the end user. The resultant ADTFRC protocol is implemented in NS-2 simulator, and the performance of ADTFRC is extensively evaluated.

Our results show that, without compromising the TCP-Friendliness property, ADTFRC outperforms TFRC and TCP NewReno with ELFN support in terms of throughput, packet loss ratio and smoothness in rate adaptation behavior. By using real MPEG-4 video traces to evaluate the streaming quality at user level, we demonstrate that ADTFRC significantly improves the streaming quality of TFRC and TCP NewReno with ELFN support.

The remainder of the paper is organized as follows: Section 2 provides an overview of our ADTFRC design. Section 3 describes the detection design. Section 4 presents the ADTFRC protocol implementation issues together with the ALF and partial reliability design. Section 5 is performance evaluations and Section 7 concludes the paper.

\section{Design Goal of ADTFRC Protocol}

The design of ADTFRC relies on the ideal rate adaptation behavior with full knowledge of the network states in ad hoc networks. We now define the network states to be distinguished and the ideal rate adaptation policies to be applied in each state. CONGESTION $(C O N G)$ : We define congestion in ad hoc networks as the signal that the offered load 
exceeds the network capacity. When congestion occurs, usually there are queue building up and the network throughput is reduced becaused of excessive contention delays and collision losses. To deal with congestion, the transport protocol should reduce the sending rate, react the same as the standard TFRC.

CHANNEL_ERR (CHERR): The receiver should not treat random packet loss as congestion event, instead of slow down, the sender should calculate the sending rate as normal.

ROUTE_CHANGE (RTCHG): Because the delivery path between two end hosts can change from time to time, disconnection may happen and it may be too transient to incur retransmission timeout. In this case, the receiver experiences a short burst of out-of-order packet delivery or packet losses. The receiver, again, should not treat it as congestion, instead, the sender should keep the streaming rate unchanged in the next RTT period, waiting for the receiver to feedback more measurement statistics for the new path.

DISCONNECTION (DISC): When the delivery path is disconnected for a long time so that a retransmission timeout happens, the sender should freeze the current congestion window and the retransmission timer instead of exponentially slow down and back off. The sender then performs a periodic probing to prepare for resuming the transmission once a new path is established. When the new path is established, the actions in the case of RTCHG are followed. We notice that such a probing technique is also proposed in [1][2].

So far, we have proposed four network states that need to be identified. In the next section, we will present a multi-metric joint detection algorithm to reliably detect the four network states by using noisy end-to-end measurements. The detection algorithm is implemented at the receiver side, which periodically updates the sender with its current network state estimation through ADTFRC feedback packets.

\section{Detection via Multiple Metrics}

\subsection{Devising End-to-End Metrics}

End-to-end measurement has been widely used in transport protocols. In TCP, the round trip time (RTT) is maintained by the sender to calculate the retransmission timeout. Previous work uses delay related metrics to measure the congestion level of the network. For example, [2] and [8] use inter packet arrival delay, and [9] uses RTT to estimate the expected throughput. A challenge in ad hoc networks is that packet delay is not only influenced by network queue length, but it is also susceptible to other conditions such as random packet loss, routing path oscillations, MAC layer contention and etc. These conditions make such measurement highly noisy. Rather than pursuing any single metric that is robust to all dynamics of the network, we devise four end-to-end metrics that tend to be influenced by different conditions so that the noise independence among them can be exploited by multi-metric joint identification.

Inter-packet delay difference (IDD): IDD measures the delay difference between a pair of consecutive packets. It measures the congestion level along the forwarding delivery path by directly sampling the transient queue size variations in the intermediate nodes. However, in an ad hoc network, there are still a number of situations in which 
$I D D$ values might give an incorrect estimation of congestion. For example, IDD can be influenced by non-congestion conditions such as mobility induced out-of-order packet delivery. We therefore introduce an additional metric $S T T$ in the following.

Short-term throughput (STT): STT is a metric used together with IDD to identify network congestion. STT provides observations within a time interval $T$, and it is less sensitive to short term out-of-order packet delivery than $I D D$. Therefore, $S T T$ is more robust to transient route changes, which can happen very frequently in ad hoc networks. However, using STT alone to detect network congestion can be susceptible to measurement noise introduced by bursty channel error, network disconnections or altering source rate. Therefore, we combine STT and IDD to jointly detect the network congestion. Aside from the IDD and STT which are related to congestion, we also consider the following two metrics for non-congestion state identification.

Packet out-of-order delivery ratio (POR): A packet is counted as being out-of-order if it arrives after a packet that was sent later than it (by the same sender). The receiver records a maximum sending time $T_{\text {maxtx }}$ for all the received packets since the establishment of the connection. Every received packet that has a sending time-stamp less than $T_{\operatorname{maxt}}$ is added into POR. POR is intended to indicate a route change event. During the route change period, multiple delivery paths exist. Packets along the new path may catch up, and those along the old path are then delivered out-of-order.

Packet loss ratio $(P L R)$ : Within each time interval $[t, t+T]$, we compute this metric as the number of missing packets in the current receiving window. PLR is used to measure the intensity of channel error.

\subsection{Detecting the Network States}

In the previous section we describe network states that are important for improving TFRC performance in ad hoc networks, and also metrics that can be measured end-toend. In this section, we study the identification of these states by using the above four metrics. Unless explicitly specification, the default settings for all the simulation results shown in this section are as follows: We use the NS-2 simulator with CMU wireless extension modules. 30 wireless nodes roam freely in a $400 m \times 800 m$ topology following a random waypoint mobility pattern, in which the pause time is zero so that each node is constantly moving. The wireless link bandwidth is $2 \mathrm{M}$ bps. IEEE 802.11 and Dynamic Source Routing (DSR, see [1]) are used as MAC and routing layer protocols respectively. One TFRC flow is created with equal packet size 1000 bytes. To introduce congestion, three competing UDP/CBR flows, each with source rate $180 \mathrm{~K}$ bps, are created within the time intervals of $[50,250],[100,200]$ and $[130,170]$ respectively. The throughput for each UDP flow is $180 \mathrm{Kbps}$. The simulations last for 300 seconds.

Detecting Congestion. To study the relationship between network congestion and IDD/STT, we simulate both static and mobile scenarios. A TFRC flow and three competing UDP/CBR flows are introduced in each simulation and the network is expected to become congested as it becomes overloaded. The first two figures of Fig. 11 show the simulation results. The first is for the static case without channel errors, and the second is for the mobile case in which node mobility speed is $5 \mathrm{~m} / \mathrm{s}$ and channel error ratio is $5 \%$. In both figures, we plot the measured IDD/STT values with respect to the instantaneous 

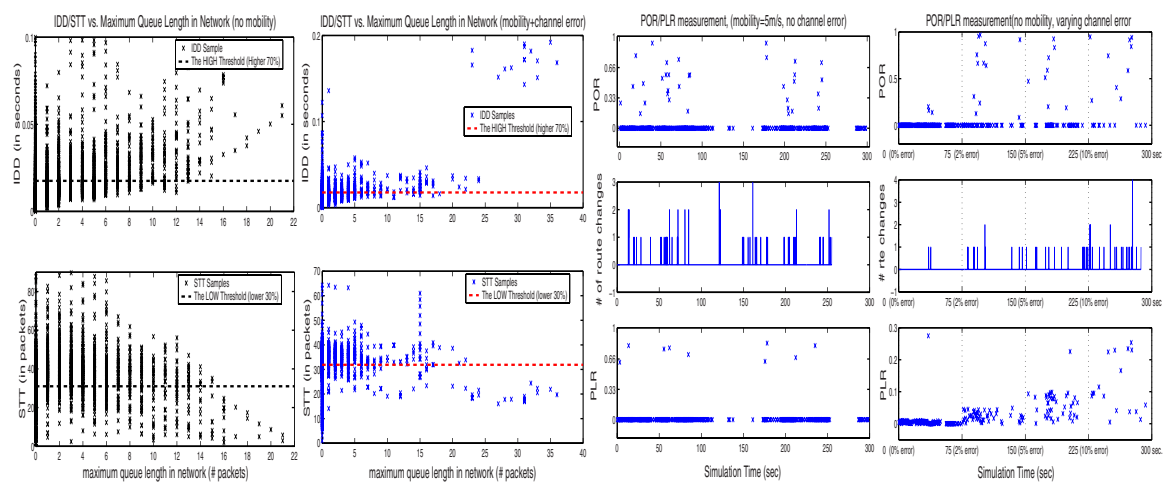

Fig. 1. End To End Metric Measurement. Left two: the IDD and STT measurement w.r.t. instantaneous maximum queue occupation of all wireless nodes in the network. First figure shows simulation with static nodes; second figure shows simulation with mobile nodes $(5 \mathrm{~m} / \mathrm{s})$. Right two: POR and PLR measurements w.r.t. the number of route changes. The third figure shows simulation with mobile nodes $(5 \mathrm{~m} / \mathrm{s})$, no channel error. The fourth one shows simulation with static nodes, progressively increasing channel error $(0 \%, 2 \%, 5 \%, 10 \%)$ during the entire run.

maximum buffer occupation of all nodes in the network, which reflects the network congestion level at the sampling time instance. 3

In Fig 1, we observe that when the maximum network queue size exceeds half of the buffer capacity (25 packets), IDD is clearly high and STT is clearly low. We formalize such an observation by defining a value to be HIGH and LOW if it is within the top and bottom $30 \%$ of all samples respectively 4 . However, when the network queue size is small (non-congestion case), both IDD and STT vary from LOW to HIGH, with the majority of IDD samples being not HIGH and STT samples not LOW. In the left two figures of Fig. 1, when node mobility is present, the two metrics become much more noisy in non-congestion state (i.e., small network queue).

In the single metric-based detection using either IDD or STT, the noise reduces the accuracy significantly when the network is not congested, especially in scenarios with mobility and channel errors. However, in the proposed joint detection approach, we can use both metrics to verify each other to improve the accuracy. Specifically, a congestion state is identified when IDD is HIGH and STT is LOW, otherwise it is identified as a non-congestion state. The following shows why the multi-metric approach has better detection accuracy than the single metric approach.

When the network is congested, let $P_{1}$ and $P_{2}$ be the probability that IDD is HIGH and STT is LOW respectively. The single metric accuracy is $a c c_{i d d}($ cong $)=P_{1}$ and $a c c_{s t t}($ cong $)=P_{2}$. For the multiple metric case, $a c c_{m u l t i}(c o n g)=P_{1} \cdot P_{2}$. Since the simulations show that $P_{1} \simeq P_{2} \simeq 1$ (see left two figures of Fig. 1), these three

\footnotetext{
${ }^{3}$ The maximum buffer size for each node is 50 packets in our simulations.

${ }^{4}$ This threshold was determined empirically from simulation results and real testbed measurements [15].
} 
accuracies are roughly equal in congestion state. On the other hand, when the network is not congested, let $P_{1}^{\prime}$ and $P_{2}^{\prime}$ be the probability that IDD is still HIGH and STT is still LOW. Similarly, we have $a c c_{i d d}($ non_cong $)=1-P_{1}^{\prime}$, acc stt $($ non_cong $)=1-P_{2}^{\prime}$ and $a c c_{\text {multi }}($ non_cong $)=1-P_{1}^{\prime} \cdot P_{2}^{\prime}$. Since each noise probability, $0<P_{1}^{\prime}, P_{2}^{\prime}<1$, is non-negligible, multiple metrics thus achieve higher accuracy. Combining these two cases, multi-metric identification improves the accuracy in non-congestion states while maintaining a comparable level of accuracy in the congestion state. Therefore, it achieves better identification performance over a variety of network conditions.

The key insight here is that in the non-congestion state, IDD and STT are influenced differently by various network conditions, such as route change and channel error; while in congestion state, they are both dominated by prolonged queuing delay. Thus, the two noise probability $P_{1}^{\prime}$ and $P_{2}^{\prime}$ become largely independent. Effective verification across multi-metrics is possible as long as these conditions do not co-exist during the measurement time interval. Although this joint identification technique cannot achieve perfect accuracy, it does increase the accuracy significantly as we show in Section 3.3

Detecting Non-congestion States. If the network state is not congestion, we next seek to detect whether it is RTCHG or CHERR. The third figure of Fig 1 shows data from a simulation run with node mobility speed being $5 \mathrm{~m} / \mathrm{s}$. A single TFRC flow is created without any competing flows that might cause network congestion. We plot POR and $P L R$ sample values together with the number of route changes in the forwarding path over time. A clear correlation is seen between route change events and bursts of high POR measurement. During the changing period, packets arrive at the receiver from multiple paths and consequently may lose their ordering. Although not all route changes result in out-of-order packet delivery, we only count those observable changes, which would have an impact upon TFRC. POR can be used to identify RTCHG state and PLR can be used for CHERR state.

Moreover, since there is no congestion or channel errors in these simulations, PLR remains stable with a few significant outliers. These anomalies correspond to situations in which packets along the old path are excessively delayed or lost.

In the simulation shown in the fourth figure of Fig 1, nodes are stationary and four channel error rates $(0 \%, 2 \%, 5 \%$ and $10 \%)$ are introduced into four identical time intervals (75 seconds). In this case, packet loss is proportional to the channel error rate and the $P L R$ gradually increases as the channel error rate increases. Note that a high channel error rate can also create route change in the network that will in turn result in bursts of high $P O R$ measurements. The routing layer interprets any MAC-layer transmission failures (in this case, channel error) as a sign of a broken link and consequently seek to repair/re-establish the delivery path, which may cause route changes.

In conclusion, a burst of high POR sample values is a good indication of a route change and a high $P L R$ is a good indication of a high rate of channel error. It should be noted that the network may be both in a state of high channel error and route change, which can be identified by high values in both $P L R$ and $P O R$.

We next consider disconnection. Disconnection happens when packet delivery is interrupted for non-congestion reasons for long enough to trigger a retransmission timeout at the sender. Multiple network conditions can trigger such a timeout at the sender 
including frequent route changes, heavy channel error, and network partition after mobility. If the timeout is triggered by congestion, then previous state feedback should reflect the transient queue build up period by increasing IDD and STT measurement at the receiver; if not, the timeout was due to non-congestion conditions in the network. Therefore, a DISC state is identified at the sender if the current state estimation is non-congestion when retransmission timeout is triggered.

Table 1. Metrics patterns in 5 network states. High: top 30\% values; Low: bottom $30 \%$ values; '*': do not care

\begin{tabular}{|c|c|c|c|}
\hline & $I D D$ and $S T T$ & $P O R$ & $P L R$ \\
\hline CONG & (High, Low) & $*$ & $*$ \\
\hline RTCHG & NOT (High, Low) & High & $*$ \\
\hline CHERR & NOT (High, Low) & $*$ & High \\
\hline DISC & $(*, \approx 0)$ & $*$ & $*$ \\
\hline NORMAL & \multicolumn{2}{|c|}{ default } \\
\hline
\end{tabular}

Table 1 summaries the metrics patterns in the four network states. Later on we show that such an identification method, combined with a simple sample classification technique, achieves an accuracy above $80 \%$ on average in all simulations scenarios.

\subsection{Detection Accuracy}

We now study the accuracy of congestion identification using the RSD technique. In particular, we compare the single-metric (using only IDD or STT) and multiple-metric (using both) approaches. We run two sets of simulations under non-congested and congested cases (Figure 2). In the first non-congested case, a single TFRC flow is created within the topology. In the second congested case, two competing UDP flows are created as before. In both cases, $1 \%$ random channel error is introduced and the mobility speed varies from 0 to $20 \mathrm{~m} / \mathrm{s}$. We repeat simulations 50 times at each speed to reduce the impact of random topology factors.

During the simulation, upon each packet loss, we compare the identified network state and the actual network state to determine the accuracy of detection 5 In particular, if a packet is lost due to network congestion, but the algorithm gives non-congestion estimation, we count it as an incompatible error because this error in detection (and only this one) causes ADTFRC to be more aggressive than a TCP-friendly flow and consequently TCP-incompatible.

Figure [2]shows the percentage of inaccurate identification in both cases. In the single TFRC flow case (the left figure), mobility and channel errors are the dominant reasons for packet loss. The increase in mobility speed reduces the accuracy of the single-metric identification quickly. However, the multi-metric approach results in only $10 \%$ to $30 \%$

\footnotetext{
${ }^{5}$ The real network state is obtained by a global monitor implemented in NS-2 simulator. See [5] for implementation details.
} 

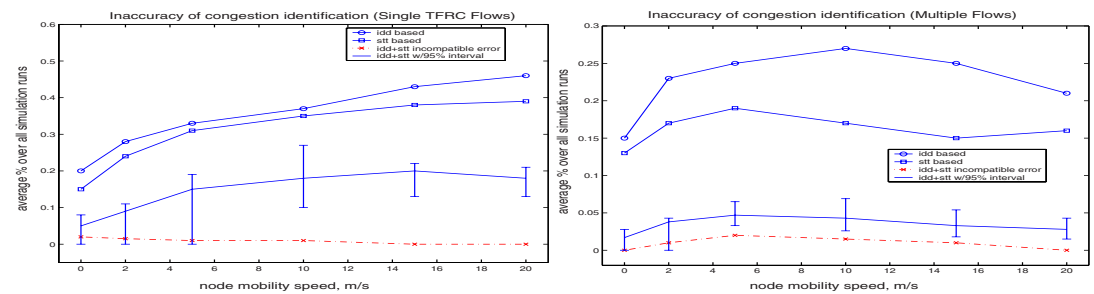

Fig. 2. Identification Accuracy. Left: Percent of inaccurate identifications in a non-congested case, Right: Inaccuracy ratio in a congested case

inaccurate identification. This is achieved by the cross verification between IDD and STT measurements to eliminate false congestion alarms. Meanwhile, the incompatible error remains less than $2 \%$.

In the multi-flow cases (the right figure), congestion happens more frequently. For multi-metric identification, more than $95 \%$ accuracy is observed in all simulations with less than $2 \%$ incompatible errors. For the single metric approach, accuracy is only about $70 \%$ to $80 \%$.

In summary, we have demonstrated that multiple metrics combined with RSD is a feasible approach to detect network events by end-to-end measurements only.

\section{ADTFRC Protocol Design and Implementation}

We now incorporate the design of Sections 2 and 3 in our ADTFRC protocol to improve the performance of TFRC in ad hoc networks.

\subsection{Adaptive Rate Adaptation}

ADTFRC seeks to maintain backward compatibility with conventional TFRC. It uses identical connection establishment and connection teardown processes. It estimates the RTT and derives the sending rate in the same way with TFRC. To improve the performance of TFRC in ad hoc networks, ADTFRC makes several extensions at both the sender side and receiver side.

Upon each packet arrival at the receiver, besides the normal operations, values for the four previously discussed metrics are calculated and network states are estimated. In ADTFRC, the congestion probability is calculated based on the outcomes of our multi-metric identification instead of the packet loss events. The receiver then passes this congestion frequency measurement together with state estimations, i.e., CONG, CHERR and RTCHG, to the sender in every feedback packet. Besides the regular feedback of each RTT, the receiver generates Urgent state update packet as soon as a congestion event is detected and fedback to the sender immediately. The sender maintains the most recently received state report, and proceeds with normal TFRC operations until either of the following two events happen: the reception of feedback packet, or the re-transmission 


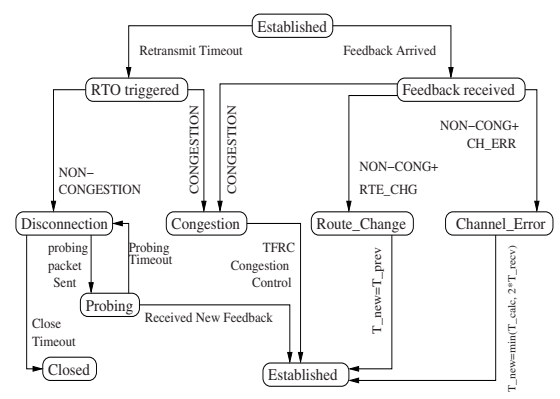

Fig. 3. ADTFRC state diagram for sender in NS-2 implementation

time out. A modified TFRC state diagram is shown in Figure 3 for the sender. The pseudo code is avaialbe in our technical report [15].

A feedback report or retransmission timeout triggers ADTFRC to take different control actions according to the current network state estimation. In particular, a probing state is introduced to explicitly handle network disconnection. When a non-congestion induced retransmission timeout occurs at the sender, ADTFRC freezes its current transmission state and enters a probing state. The sender leaves the probing state when a new acknowledgement is received or the probing is timed out The ADTFRC connection is closed after multiple probing attempts fail.

\section{Performance Evaluation}

In this section, we evaluate the performance of ADTFRC through extensive NS-2 simulations in terms of its throughput, rate adaptation behavior as well as the application level quality perceived by the end user.

\subsection{Throughput Improvement}

In the throughput evaluation part, we compare it to TFRC and TCP with ELFN [1] support. Instead of using end-to-end measurements, TCP ELFN collects link state information directly from the network and is expected to be more accurate. It is used as a reference system; a throughput close to ELFN indicates the effectiveness of ADTFRC.

Figure 4 shows the single flow throughput of ADTFRC, TFRC and TCP-NewReno with ELFN support. The simulation parameters for TFRC flows are set as described in section three, and for TCP ELFN flow, we set the packet size to be 1000 bytes and maximum window size to be 8 packets. In all three cases, ADTFRC provides significantly better throughput than TFRC. When nodes are mobile, ADTFRC achieves a throughput improvement from $100 \%$ to $800 \%$ over TFRC. Furthermore, it is surprising to see that ADTFRC out-performs TCP+ELFN even in a static network where the mobility speed is zero. The reason is because the ACK packet traffic on the reverse path is much heavier in

\footnotetext{
${ }^{6}$ A similar probing mechanism was proposed by [1]
} 

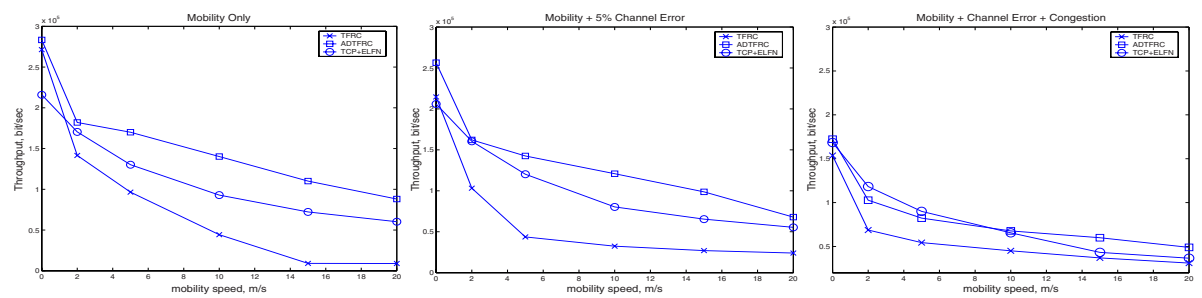

Fig. 4. Performance Improvement of ADTFRC. From left to right:1)mobility only, 2)mobility+5\% channel error, 3) mobility $+5 \%$ channel error +3 competing UDP/CBR flows

TCP+ELFN than in ADTFRC. Due to the broadcast nature of the wireless link, such ACK flows contend for the channel access with forwarding data flows, introducing additional delay in RTT and resulting in throughput decrease.

\subsection{Rate Adaptation}

We further measure the rate oscillations experienced at the receiver for each of these three protocols. To effectively support best-effort multimedia streaming, dramatic rate variations are highly undesirable.
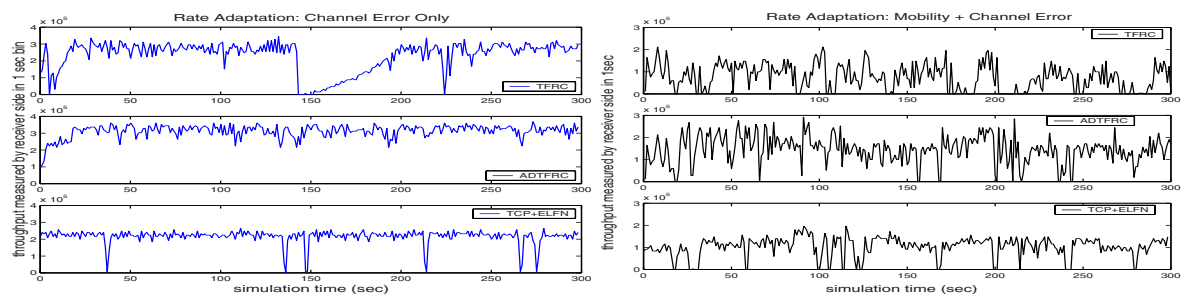

Fig. 5. Smoothness of Rate Adaptation. From left to right:1)Single flow in static ad hoc network, $2 \%$ random channel error. 2) Single flow with node mobility $5 \mathrm{~m} / \mathrm{s}, 2 \%$ random channel error.

In Figure 5 we show the throughput fluctuations at the receiver side in two environment settings. The left figure is for the simulation in static network, with $2 \%$ random channel error. It shows that the ADTFRC (the middle one) maintains a more stable transport rate.

When mobility and channel error are both introduced as shown in the right one of Figure 5, the rate variation of ADTFRC becomes much larger. However due to a probing mechanism of ADTFRC, the transmission interruptions are much smaller than the TFRC flow. For TCP+ELFN flow, although its disconnection period is also short, due to its aggressive bandwidth probing mechanism, it again encounters more frequent interruptions than ADTFRC. 


\subsection{Application Layer Quality}

To evaluate the quality improvement perceived by an end host, we use an application layer metric, client starvation time that is defined in [26]. This metric characterizes the situation when the client experience a freezing motion or frame skip during the streaming. We use the StarWar video trace [25] encoded with 70Kbps average source rate in MPEG-4 format. The application layer framing and partial reliability is enabled at the two end hosts. The implementation details are again referred to the technical report [15].

Table 2. The (aggregated) client starvation time (in seconds) for single MPEG-4 streaming and two simultaneous flows.

\begin{tabular}{|c|l|l|l|l||l|l|l|l|l|}
\hline & \multicolumn{3}{c||}{ Single Flow } & \multicolumn{3}{c|}{ Aggregated for Two Flows } \\
\hline & $0 \mathrm{~m} / \mathrm{s}$ & $2 \mathrm{~m} / \mathrm{s}$ & $5 \mathrm{~m} / \mathrm{s}$ & $10 \mathrm{~m} / \mathrm{s}$ & $0 \mathrm{~m} / \mathrm{s}$ & $2 \mathrm{~m} / \mathrm{s}$ & $5 \mathrm{~m} / \mathrm{s}$ & $10 \mathrm{~m} / \mathrm{s}$ \\
\hline TFRC & 14 & 69 & 102 & 178 & 31 & 308 & 1083 & 4032 \\
\hline ADTFRC & 2 & 15 & 42 & 58 & 4 & 137 & 216 & 367 \\
\hline TCP-ELFN & 6 & 17 & 39 & 67 & 14 & 187 & 831 & 6873 \\
\hline
\end{tabular}

Observe from Table 2 the streaming quality measured by client starvation time is greatly improved by ADTFRC. Compared with standard TFRC or TCP+ELFN, the improvement is especially significant in scenarios when multiple streaming flows run in a mobile network. A detailed trace analysis is available in our technical report [15].

\section{Conclusion}

In this paper, we explore an end-to-end approach to design a TCP-friendly transport protocol, ADTFRC, to improve the performance of rate adaptation of TFRC in mobile ad hoc network. ADTFRC uses multiple metrics to jointly detect network states in the presence of measurement noises, so that the sending rate can be adjusted accordingly. We also propose the application layer framing to further enhance the streaming quality perceived by the end user. Simulations show that ADTFRC can significantly improves the performance of real-time video streams in a TCP friendly way.

\section{References}

1. G. Holland and N. Vaidya, "Analysis of TCP performance over mobile ad hoc networks," MOBICOM'99.

2. P. Sinha, N. Venkitaraman, R. Sivakumar and V. Bharghavan, "WTCP: A reliable transport protocol for wireless wide-area networks," MOBICOM'99.

3. H. Balakrishnan, S. Seshan, E. Amir, and R. Katz, "Improving TCP/IP performance over wireless networks," MOBICOM'95.

4. H. Balakrishnan, and R. Katz, "Explicit loss notification and wireless web performances," Globecom'98. 
5. Zhenghua Fu, Xiaoqiao Meng, Songwu Lu, "How bad TCP can perform in wireless ad hoc network" IEEE ISCC (IEEE Symposium on Computers and Communications) 2002, Italy, July 2002.

6. J. Monks, P. Sinha and V. Bharghavan, "Limitations of TCP-ELFN for ad hoc networks," MOMUC'OO.

7. M. Gerla, K. Tang, and R. Bagrodia, "TCP performance in wireless multihop networks," WMCSA'99.

8. S. Biaz and N.H. Vaidya, "Distinguishing congestion losses from wireless transmission losses" IEEE 7th Int. Conf. on Computer Communications and Networks, October 1998.

9. L. Brakmo, S. O’Malley, and L. Peterson "TCP Vegas: New techniques for congestion detection and avoidance" ACM SIGCOMM 1994

10. V. Jacobson and M.J. Karels, "Congestion Avoidance and Control" ACM Sigcomm 1988

11. M. Allman, V. Paxson, and W. Stevens, "TCP Congestion Control" RFC 2581 April 1999.

12. S. Biaz, N. Vaidya, "Discriminating congestion losses from wireless losses using inter-arrival times at the receiver" IEEE ASSET 1999

13. A. Bakre and B. Badrinath, "I-TCP:indirect TCP for mobile hosts", Proc. 15th International Conf. on Distributed Computing Systems (ICDCS) May 1995

14. A. Bakre and B. Badrinath, "Implementation and performance evaluation of Indirect TCP" IEEE Trans. Computers, Vol. 46, March 1997

15. Z. Fu, X. Meng, S. Lu, "TCP Friendly Rate Adaptation for Multimedia Streaming in Mobile Ad Hoc Networks", Technical Report of Computer Science Department, UCLA, 2003.

16. S. Floyd, M. Handley, J. Padhye and J. Widmer, "Equation-Based Congestion Control for Unicast Applications" In Proceedings of ACM Sigcomm 2000

17. M. Handley, S. Floyd, J. Padhye and J. Widmer, "TCP Friendly Rate Control (TFRC): Protocol Specification", RFC 3448, January 2003.

18. D. Bansal and H. Balakrishnan, "Binomial Congestion Control Algorithms." In Proceedings of IEEE Infocom, 2001.

19. D. Bansal, H. Baladrishnan, S. Floyd and S. Shenker, "Dynamic Behavior of SlowlyResponsive Congestion Control Algorithms", In Proceedings of ACM Sigcomm 2001

20. H. Schulzrinne, S. Casner, R. Frederick, V. Jacobson, "RTP: A Transport Protocol for RealTime Applications", RFC 1889, 1996

21. S. Raman, H. Balakrishnan, M. Srinivasan, "ITP: An Image Transport Protocol for the Internet", IEEE/ACM Trans. on Networking, June, 2002.

22. M. Feamster and H. Balakrishnan, "Packet Loss Recovery for Streaming Video", 12th International Packet Video Workshop, Pittsburgh, PA, April 2002

23. J. Liu and S. Singh. "ATCP:TCP for mobile ad hoc networks", IEEE Journal on Selected Areas in Communiations, 19(7):1300-1315 July 2001

24. F. Wang and Y. Zhang, "Improving TCP performance over mobile ad-hoc networks with outof-order detection and response", In Proceedings of the third ACM international symposium on Mobile ad hoc networking and computing, 2002, Lausanne, Switzerland.

25. F. Fitzek and M. Reisslein, "MPEG-4 and H.263 Video Traces for Network Performance Evaluation", IEEE Network, Vol.15 No.6, November 2001. Video traces available from the website http://www-tkn.ee.tu-berlin.de/fitzek/TRACE/pub.html

26. F. Fitzek, M. Reisslein, "A Prefetching Protocol for Continuous Media Streaming in Wireless Environments", IEEE Journal on Selected Areas in Communications (Special Issue on Mobility and Resource Management in Next Generation Wireless Systems), Vol.19, No.6 Pages 2015-2028, October 2001. 